\title{
Efeito da fisioterapia sobre os parâmetros ventilatórios e a dinâmica tóraco-abdominal de crianças respiradoras bucais
}

\author{
Effects of the physical therapy on ventilatory parameters and \\ thoracoabdominal dynamics of mouth breathing children
} Fabiana dos Santos Ferreira', Priscila Weber², Eliane Castilhos Rodrigues Corrêa ${ }^{3}$,
Jovana de Moura Milanesi ${ }^{4}$, Gracieli da Silva Borin ${ }^{4}$, Mônica Falk Dias ${ }^{5}$

RESUMO I Este estudo objetivou avaliar o efeito da fisioterapia nos parâmetros ventilatórios e na dinâmica tóraco-abdominal de crianças respiradoras bucais. Dez crianças respiradoras bucais foram submetidas a um programa de reeducação respiratória e correção postural durante 20 sessões de exercícios de alongamento e fortalecimento muscular sobre a bola suíça, estimulação diafragmática e alongamento dos músculos acessórios da inspiração. Os dados coletados foram as medidas de pressões inspiratórias e expiratórias máximas (PIM e PEM), capacidade inspiratória (Cl), pico de fluxo expiratório (PFE), mobilidade torácica por meio do coeficiente respiratório ( $\mathrm{Cr}$ ) nos níveis axilar ( $\mathrm{CrA}$ ), xifoide ( $\mathrm{CrX}$ ) e umbilical (CrU) e análise biofotogramétrica do ângulo de Charpy. Estes foram verificados antes e após 10 e 20 atendimentos. Os dados foram submetidos a uma análise de variância (ANOVA) e, nas diferenças encontradas entre as médias, aplicou-se o Teste de Tukey com nível de significância de 5\% ( $p<0,05)$. Houve melhora para as medidas referentes ao ângulo de Charpy ( $p=0,048), C l(p=0,037)$, PFE (0,002), PIM (0,007), CrA ( $p=0,008)$ e CrX ( $p=0,041)$. A PEM e o CrU, apesar da melhora nos valores absolutos, não apresentaram resultados estatisticamente significativos. 0 programa de fisioterapia respiratória e postural produziu efeitos positivos sobre a função ventilatória de crianças respiradoras bucais.

Descritores I fisioterapia; respiração bucal; fotogrametria.

\begin{abstract}
I This study aimed to evaluate the effects of the physiotherapy on the ventilatory parameters and thoracoabdominal dynamics of mouth breathing children. Ten mouth breathing children were undergone to 20 sessions of respiratory reeducation and postural correction through stretching and strengthening exercises on Swiss ball, diaphragmatic stimulation and stretching of the inspiratory accessory muscles. The data collected were measurements of the maximum inspiratory and expiratory pressures (MIP and MEP), inspiratory capacity (IC), peak expiratory flow (PEF), the chest wall mobility measured by the respiratory coefficient (Rc) at the levels of the axillary (RcA), xiphosternal (RcX) and umbilical (RcU) regions and biophotogrametry analysis of the Charpy angle. These were measured before and after 10 and 20 sessions. A 1-way repeated measures analysis of variance (ANOVA) was used to determine differences in variables among the three evaluations. Post-hoc comparisons were conducted with the Tukey's Test. It was considered a significance level of $5 \%(p<0.05)$. There were improvements on Charpy angle $(p=0.048)$, IC ( $p=0.037)$, PEF (0.002), MIP ( $p=0.007)$, RcA $(p=0.008)$ and RcX ( $p=0.041)$ measures. Although MEP and $\mathrm{RcU}$ improved their absolute values, the results were not statistically significant. The program of respiratory physiotherapy and postural correction produced positive effects on the ventilatory function of mouth breathing children.
\end{abstract}

Keywords I physical therapy; mouth breathing; photogrammetry.

\footnotetext{
Estudo desenvolvido no Laboratório de Motricidade Orofacial da Universidade Federal de Santa Maria (UFSM) - Santa Maria (RS), Brasil. 'Fisioterapeuta; Mestranda do Programa de Pós-graduação em Distúrbios da Comunicação Humana da UFSM - Santa Maria (RS), Brasil. 2Fisioterapeuta; Bolsista da Coordenação de Aperfeiçoamento de Pessoal de Nível Superior (CAPES); Mestranda do Programa de Pósgraduação em Distúrbios da Comunicação Humana da UFSM - Santa Maria (RS), Brasil.

${ }^{3}$ Fisioterapeuta; Doutora em Biologia Buco-dental pela Universidade Estadual de Campinas (UNICAMP); Professora do Departamento de Fisioterapia e Reabilitação e do Programa de Pós-graduação em Distúrbios da Comunicação Humana da UFSM - Santa Maria (RS), Brasil. ${ }^{4}$ Fisioterapeuta; Mestre em Distúrbios da Comunicação Humana pela UFSM - Santa Maria (RS), Brasil.

${ }^{5}$ Fisioterapeuta pela UFSM - Santa Maria (RS), Brasil.
} 


\section{INTRODUÇÃO}

A Síndrome do Respirador Oral (SRO) é a situação na qual a respiração oral de suplência é estabelecida ${ }^{1}$. Como consequência, alterações do complexo craniofacial podem estar presentes, influenciando negativamente as funções estomatognáticas e causando desequilíbrios posturais e respiratórios por uso excessivo da musculatura acessória da respiração $\mathrm{o}^{2-4}$. Além dos prejuízos respiratórios e posturais, a respiração oral pode influenciar adversamente a qualidade de vida, principalmente por afetar o sono, o que prejudica o aprendizado e a cognição $0^{5,6}$.

Ao respirar pela boca, a criança apresenta uso anormal dos músculos respiratórios, comumente associado à fadiga $^{7}$. Com isso, a capacidade inspiratória, as pressões respiratórias máximas e a mobilidade torácica podem ser alteradas devido a mudanças estruturais na caixa torácica $^{8-11}$. Alguns estudos já demonstraram a interferência positiva do estímulo diafragmático, diagonais de Kabat e Reeducação Postural Global (RPG) na condição respiratória, com aumento das pressões respiratórias máximas e expansão torácica em pessoas hígidas ${ }^{12,13}$.

Sendo assim, um programa fisioterapêutico que melhore a mecânica ventilatória proporciona ao respirador bucal a obtenção de parâmetros ventilatórios próximos aos valores de normalidade ${ }^{14}$. O presente estudo se propôs a avaliar o efeito de um programa de fisioterapia, com ênfase na reeducação muscular respiratória e correção postural, nos parâmetros ventilatórios e na dinâmica tóraco-abdominal de crianças respiradoras bucais.

\section{MATERIAIS E MÉTODOS}

O projeto deste estudo foi aprovado pelo Comitê de Ética em Pesquisa, conforme parecer número 0006.0.243.000-07, reconhecido pela Comissão Nacional de Ética em Pesquisa (CONEP). Trata-se de um estudo experimental, de caráter prospectivo, com análise quantitativa dos dados.

Para serem incluídas na pesquisa, as crianças deveriam ter entre 8 e 12 anos de idade; diagnóstico otorrinolaringológico de respiração bucal; e apresentar o Termo de Consentimento Livre e Esclarecido assinado pelos pais e/ou responsáveis. Os critérios de exclusão foram presença de comprometimento neuropsicomotor e/ou qualquer limitação física que impedisse a realização do tratamento.
Participaram deste estudo dez crianças respiradoras bucais, sendo seis meninos e quatro meninas, com idade de 10 $\pm 2,1$ anos. Sessenta por cento delas apresentaram rinite alérgica, $30 \%$ hipertrofia de adenóide e 10\% respiração bucal persistente após adenoidectomia recente como fatores etiológicos para a sua respiração bucal. Tais fatores foram diagnosticados por avaliação otorrinolaringológica em exames de nasofibrolaringoscopia e oroscopia.

\section{Procedimentos}

$\mathrm{Na}$ avaliação inicial verificaram-se a pressão inspiratória (PIM) e expiratória máxima (PEM), capacidade inspiratória (CI), o pico de fluxo expiratório (PFE) e a mobilidade tóraco-abdominal. Todas as mensurações foram realizadas por um único examinador, o qual não participou da aplicação do protocolo de tratamento. As ponderações foram obtidas com a criança confortavelmente sentada, estando os braços ao longo do corpo e pés apoiados no chão, visto que esta posição favorece a maior expansão da caixa torácica e do pulmão ${ }^{15}$.

Registraram-se as medidas de PIM e a PEM a fim de se avaliar a força dos músculos inspiratórios e expiratórios. Elas foram coletadas em um manovacuômetro analógico da marca SUPORTE, com intervalo operacional de $\pm 150 \mathrm{cmH}_{2} \mathrm{O}$. Para a coleta, foi utilizado um clipe nasal. A manobra de PIM foi feita a partir do volume residual pulmonar (VR) e a da PEM a partir da capacidade pulmonar total (CPT). Foram anotadas três medidas, sendo escolhida a pressão de maior valor ${ }^{16-19}$.

Para a avaliação da capacidade inspiratória foi utilizado um inspirômetro de incentivo a volume da marca Voldyne $^{19}$. O pico de fluxo expiratório foi aferido pelo instrumento Peak Flow Meter da marca Assess ${ }^{20}$. Realizaram-se três medidas para cada avaliação, sendo escolhida a maior dentre elas.

A averiguação da dinâmica tóraco-abdominal foi feita pela cirtometria torácica, sendo utilizada uma fita métrica da marca WISO. Foram medidos os perímetros em três regiões do tórax: axilar, ao nível da terceira costela; xifoide, ao nível da sétima cartilagem costal; e umbilical, ao nível das últimas costelas. As medidas foram realizadas durante a respiração em volume corrente e na inspiração e expiração máximas. Para aferir a dinânima tóraco-abdominal, considerou-se a diferença entre as medidas de inspiração e expiração máximas, denominada coeficiente respiratório $(\mathrm{Cr})^{21}$.

O ângulo de Charpy foi avaliado na postura ortostática por meio de registro fotográfico, com análise 
biofotogramétrica no programa SAPO, um software de avaliação postural gratuito que permite a medida de ângulos e distâncias da postura corporal ${ }^{22}$. As aquisições das imagens foram feitas em uma máquina fotográfica digital (Sony Cybershot 7.2 megapixels) posicionada em um tripé localizado a 2 metros da criança e a 1 metro de altura do chão. Os pontos demarcados para a formação do ângulo de Charpy foram apêndice xifoide e, bilateralmente, o ângulo inferior das últimas costelas ${ }^{23}$.

As crianças foram submetidas a um Programa de Reeducação Muscular Respiratória, por meio de estimulação diafragmática e alongamento manual dos músculos acessórios da inspiração, e a exercícios para correção postural na bola suíça. Os exercícios foram realizados na posição sentada, em prono e em supino, priorizando basicamente o realinhamento da postura por meio do alongamento dos músculos anteriores e fortalecimento dos músculos posteriores do tronco ${ }^{24}$. O tratamento contou com 20 sessões semanais com duração de 60 minutos cada. Houve reavaliação das crianças depois de finalizadas a $10^{\mathrm{a}}$ e $20^{\mathrm{a}}$ sessões.

Tabela 2. Comparação dos parâmetros ventilatórios e ângulo de Charpy no pré-tratamento, após 10 sessões e após 20 sessões ( $n=10$ )

\begin{tabular}{|c|c|c|}
\hline $\begin{array}{l}\text { Parâmetros } \\
\text { ventilatórios }\end{array}$ & $\begin{array}{l}\text { ANOVA }^{\text {a }} \\
\text { (Valor } p \text { ) }\end{array}$ & $\begin{array}{l}\text { Comparações } \\
\text { múltiplas }^{b}\end{array}$ \\
\hline Ângulo de Charpy & 0,048 & $\mu_{\text {pre }} \# \mu_{\text {20sessóes }}$ \\
\hline Capacidade inspiratória & 0,037 & $\mu_{\text {pre }} \# \mu_{10 \text { sessōes }}$ \\
\hline Pico de fluxo & 0,002 & $\begin{array}{l}\mu_{\text {pre }} \# \mu_{10 \text { sessōes }} \\
\mu_{\text {pre }} \# \mu_{20 \text { sessöes }}\end{array}$ \\
\hline Pressão inspiratória máxima & 0,007 & 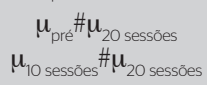 \\
\hline Pressão expiratória máxima & 0,781 & * \\
\hline CR axilar & 0,008 & $\begin{array}{l}\mu_{\text {pré }} \# \mu_{10 \text { sessōes }} \\
\mu_{\text {pré }} \# \mu_{20 \text { sessōes }}\end{array}$ \\
\hline CR xifoide & 0,041 & $\mu_{\text {pre }} \# \mu_{10 \text { sessöes }}$ \\
\hline CR umbilical & 0,253 & * \\
\hline
\end{tabular}

análise de variância; ${ }^{\circ}$ Teste de Tukey: $\mathrm{p} \leq 0,05 ; \mu_{\mathrm{pres}}$ : média das avaliações realizadas antes de iniciar o tratamento fisioterapêutico; $\mu_{10 \text { coescosoc: }}$ média das avaliaçōes realizadas após 10 sessões

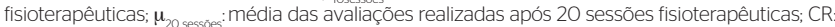
coeficiente respiratóric

*Não houve diferença estatística entre os tempos de avaliação

\section{Análise estatística}

O teste Shapiro-Wilk verificou a normalidade das variáveis. Posteriormente, os dados coletados foram submetidos a uma análise de variância (ANOVA) e, nas diferenças encontradas entre as médias, aplicou-se o Teste de Tukey, com nível de significância de 5\%.

\section{RESULTADOS}

A Tabela 1 apresenta a análise descritiva dos valores obtidos nos diferentes tempos de avaliações dos parâmetros ventilatórios e ângulo de Charpy.

Após dez sessões, houve aumento na capacidade inspiratória $(\mathrm{p}=0,05)$ e redução do coeficiente respiratório xifoide $(\mathrm{p}=0,03)$. Entre a avaliação inicial e após a $20^{\mathrm{a}}$ sessão, houve redução nos valores aferidos para o ângulo de Charpy $(\mathrm{p}=0,04)$. O pico de fluxo $(\mathrm{p}=0,02$ e $\mathrm{p}<0,01)$ aumentou e o coeficiente respiratório axilar ( $p=0,03$ e $\mathrm{p}=0,01)$ reduziu após 10 e 20 sessões. Entre as avaliações iniciais e após 20 sessões e entre 10 e 20 sessões observou-se aumento na pressão inspiratória máxima $(\mathrm{p}<0,01$ e p=0,02). Já a pressão expiratória máxima e o coeficiente respiratório umbilical, apesar de terem seus valores absolutos modificados com o tratamento, não apresentaram diferenças significativas entre as avaliações (Tabela 2).

\section{DISCUSSÃO}

O tratamento proposto proporcionou aumento considerável no volume pulmonar constatado pelo significativo incremento da capacidade inspiratória. Este achado pode ser explicado pelo crescimento da força dos músculos inspiratórios, também obtido com o tratamento.

Tabela 1. Média e desvio padrão dos parâmetros ventilatórios e ângulo de Charpy no pré-tratamento, após 10 sessões e após 20 sessões ( $\mathrm{n}=10$ )

$\begin{array}{lccc}\text { Parâmetros } & \text { Pré-tratamento } & \text { Pós 10 sessões } & \text { Pós 20 sessões } \\ \text { Ângulo de Charpy }\left(^{\circ}\right) & 86,98 \pm 07,91 & 89,34 \pm 4,54 & 91,01 \pm 2,95 \\ \mathrm{Cl}(\mathrm{mL}) & 1350,00 \pm 0,38 & 2010,00 \pm 0,90 & 20175,00 \pm 1,01 \\ \mathrm{PFE}(\mathrm{L} / \mathrm{min}) & 237,50 \pm 67,73 & 275,50 \pm 72,43 & 292,50 \pm 78,57 \\ \mathrm{PIM}\left(\mathrm{cmH}_{2} \mathrm{O}\right) & 72,00 \pm 12,30 & 74,40 \pm 9,26 & 88,00 \pm 20,02 \\ \text { PEM }\left(\mathrm{cmH}_{2} \mathrm{O}\right) & 75,00 \pm 17,48 & 78,50 \pm 15,46 & 79,00 \pm 15,05 \\ \mathrm{CR} \text { axilar }(\mathrm{cm}) & 5,60 \pm 2,34 & 3,40 \pm 1,37 & 2,95 \pm 1,61 \\ \mathrm{CR} \text { xifoide }(\mathrm{cm}) & 4,40 \pm 2,01 & 2,50 \pm 1,15 & 3,50 \pm 1,25 \\ \mathrm{CR} \text { umbilical }(\mathrm{cm}) & 4,24 \pm 2,12 & 5,65 \pm 1,49 & 5,05 \pm 1,88\end{array}$

Cl: capacidade inspiratória; PFE: pico de fluxo expiratório; PIM: pressão inspiratória máxima; PEM: pressão expiratória máxima; CR: coeficiente respiratório 
A contração efetiva destes músculos proporciona maior expansão do gradil costal, alargando o volume de ar inspirado ${ }^{10,25}$. Banzatto et al. ${ }^{19}$ verificaram aumento da capacidade inspiratória de crianças respiradoras bucais após adenotonsilectomia. Estes resultados foram atribuídos ao aumento da PIM e do perímetro abdominal observados após a cirurgia. Os achados coincidem com os do presente estudo, porém mostram que, mesmo em casos alérgicos ou quando a obstrução ainda não foi resolvida cirurgicamente, a fisioterapia pode produzir efeitos positivos na mecânica ventilatória destas crianças.

A literatura reporta o valor médio de normalidade para a PIM de $105 \pm 27 \mathrm{cmH}_{2} \mathrm{O}$ para os meninos e $71 \pm 29 \mathrm{cmH}_{2} \mathrm{O}$ para as meninas saudáveis na faixa etária de $10 \operatorname{anos}^{16,26}$. Na avaliação que antecedeu o tratamento, observou-se PIM médio de 72,0 $\pm 12,3 \mathrm{cmH}_{2} \mathrm{O}$. Apesar do gênero não ter sido considerado na interpretação dos resultados, o grupo de estudo foi composto na maior parte $(60 \%)$ por meninos, portanto os valores de PIM obtidos foram baixos comparados aos referenciais de crianças saudáveis. Pires et al. ${ }^{27}$ confirmaram valores médios de PIM significativamente menores para um grupo de crianças respiradoras orais quando comparadas a um grupo controle de crianças respiradoras nasais.

O aumento da PIM observado após o tratamento pode indicar que as crianças desenvolveram melhor uso do diafragma, o que pode ter favorecido o seu fortalecimento. Além disso, a adoção de um padrão predominantemente costo-diafragmático pode ter refletido na melhora dos valores de $\mathrm{PIM}^{28}$. Banzatto et al. ${ }^{19}$ verificaram aumento da PIM em crianças respiradoras bucais após adenotonsilectomia, sugerindo que, com a ausência do fator obstrutivo a readequação da mecânica ventilatória pode ter repercutido positivamente sobre o desempenho dos músculos inspiratórios. Em outro estudo ${ }^{18}$, com programa de biofeedback respiratório, as crianças respiradoras bucais apresentaram incremento da PIM, o que indica que melhoraram o uso do diafragma após a sua reeducação. Quanto aos valores de PEM, registrados após o tratamento, houve aumento em relação aos valores aferidos antes do tratamento, porém sem significância estatística. Em 2003, Fauroux ${ }^{16}$ definiu para os meninos na faixa etária de 10 anos, valor médio da PEM de $123 \pm 27 \mathrm{cmH}_{2} \mathrm{O}$. Considerando que $60 \%$ do grupo de estudo foi representado por meninos, observa-se que, conforme valores de referência, eles mantiveram-se abaixo da média proposta para a idade mesmo após realizarem o tratamento.

Considerando que o PFE depende do esforço do paciente durante a manobra e da força muscular respiratória ${ }^{29}$, o aumento nos valores deste parâmetro pode ser explicado pelo da PIM ao longo do tratamento. Corroborando com o raciocínio, Lima et al. ${ }^{9}$ realizaram um treino da musculatura inspiratória em crianças asmáticas e obtiveram incremento significativo nos valores de $\mathrm{PFE}$, atribuindo estes achados ao crescimento da força muscular inspiratória e alongamento adequado dos músculos respiratórios. Por outro lado, um estudo ${ }^{30}$ com crianças respiradoras bucais sobre o efeito do biofeedback respiratório associado ao padrão de quiet breathing, descrito como padrão fisiológico sem esforço, não obteve melhora do PFE.

$\mathrm{Na}$ avaliação que antecedeu o tratamento foi observado padrão respiratório com amplitude predominantemente apical. Este resultado confirma os achados de Barbiero et al. ${ }^{18}$, que também observaram padrão respiratório irregular em crianças respiradoras bucais com predominância do padrão torácico apical em 50\% da amostra estudada. Após o tratamento, elas apresentaram padrão melhor distribuído em relação ao tórax superior e ao abdômen, com preferência ao padrão costo-diafragmático. Isso indica que a terapia priorizada neste estudo, que incluía estimulação diafragmática manual e, principalmente, alongamento da musculatura acessória, pode ter proporcionado a liberação da caixa torácica, anteriormente em posição apical ${ }^{24,30,31}$. Estes resultados assemelham-se aos de Corrêa e Beézin ${ }^{24}$,que observaram a diminuição da atividade elétrica dos músculos cervicais durante a inspiração nasal após um programa de exercícios respiratórios e posturais em bola suíça. Barbiero et al. ${ }^{30}$ trataram 20 crianças respiradoras bucais funcionais durante 15 sessões diárias de biofeedback respiratório associado ao padrão quiet breathing e também obtiveram melhora da dinâmica tóraco-abdominal, cujo padrão respiratório predominantemente torácico superior passou a ser predominantemente abdominal ou misto.

O aumento do ângulo de Charpy observado após o tratamento pode ser atribuído à mudança no padrão ventilatório, uma vez que o recrutamento muscular diafragmático proporciona maior mobilidade das costelas inferiores e aumento do diâmetro transversal inferior do gradil costal ${ }^{30,31}$. Por outro lado, no estudo de Baraúna e Deloroso $^{23}$, crianças asmáticas apresentaram valores de média para o ângulo de Charpy de 96²4', enquanto o número foi $73^{\circ} 02$ em não asmáticas. Os autores destacam a importância desta medida pois a partir da alteração do ângulo outras alterações podem ocorrer, contribuindo para a instalação da má postura e suas consequências.

As técnicas de correção postural e respiratória podem influenciar positivamente o padrão de crianças respiradoras bucais. Além disso, o estímulo diafragmático, com ênfase na expansão da caixa torácica, pode 
ajudar quando há uso excessivo dos músculos acessórios do pescoço e do tórax superior ${ }^{24}$. É ainda recomendável que o padrão diafragmático não seja simplesmente demonstrado, e sim facilitado por uma readequação da mecânica tóraco-abdominal ${ }^{24,31}$ o que parece ter sido alcançado pelas crianças do estudo após a consciência do padrão diafragmático com orientação e estímulo manual conjuntamente com o relaxamento e alongamento dos músculos acessórios.

Este estudo teve como limitações a falta de um grupo controle para comparação dos resultados. Além disso, considerando o impacto negativo da respiração bucal na qualidade de vida, verifica-se também a importância de investigar o efeito do tratamento neste aspecto.

Mais estudos são necessários sobre a reeducação da mecânica ventilatória após a remoção do fator obstrutivo, pois mesmo em crianças com respiração bucal de causa orgânica não corrigida, o programa fisioterapêutico já apresentou resultados benéficos, criando expectativas promissoras quando a abordagem terapêutica for completa e multidisciplinar.

\section{CONCLUSÃO}

O programa fisioterapêutico com ênfase na readequação da mecânica ventilatória, associado aos exercícios posturais corretivos na bola suíça, desempenhou importante papel no tratamento das crianças respiradoras bucais avaliadas, uma vez que produziu efeitos positivos sobre os parâmetros ventilatórios e repercutiu favoravelmente sobre a mobilidade torácica.

\section{REFERÊNCIAS}

1. Di Francesco RC, Passerotii G, Paulucci B, Miniti A. Respiração oral na criança: repercussões diferentes de acordo com o diagnóstico. Rev Bras Otorrinolaringol. 2004;70(5):665-70.

2. Lessa FC, Enoki C, Feres MF, Valera FC, Lima WT, Matsumoto MA. Breathing mode influence on craniofacial development. Rev Bras Otorrinolaringol. 2005; 71(2):156-60.

3. Lemos CM, Wilhelmsen NS, Mion OG, Mello Júnior JF. Functional alterations of the stomatognathic system in patients with allergic rhinitis: case-control study. Rev Bras Otorrinolaringol. 2009;75(2):268-74.

4. Ferla A, Silva AMT, Correa ECR. Atividade eletromiográfica dos músculos temporal anterior e masseter em crianças respiradoras bucais e em respiradoras nasais. Rev Bras Otorrinolaringol. 2008;74(4):588-95.
5. Campanha SMA, Freire LMS, Fontes MJF. O impacto da asma, da rinite alérgica e da respiração oral na qualidade de vida de crianças e adolescentes. Rev CEFAC. 2008;10(4):513-19.

6. Camelo-Nunes IC, Solé D. Allergic rhinitis: indicators of quality of life. J Bras Pneumol. 2010;36(1):124-33.

7. Banzatto MG, Grumach AS, Mello JF Jr, Di Francesco RC. Adenotonsillectomy improves the strength of respiratory muscles in children with upper airway obstruction. Int J Pediatr Otorhinolaryngol. 2010;74(8):860-3.

8. Pires MG, Di Francesco RC, Mello JF Jr, Grumach AS. Chest modification secondary to the enlarged tonsils and adenoids. Intl Arch Otorhinolaryngol. 2007:11(2):99-105.

9. Lima EV, Lima WL, Nobre A, dos Santos AM, Brito LM, Costa MR. Inspiratory muscle training and respiratory exercises in children with asthma. J Bras Pneumol. 2008:34(8):552-8.

10. Yi LC, Jardim JR, Pignatari SS, Inoue DP. The relationship between excursion of the diaphragm and curvatures of the spinal column in mouth breathing children. J Pediatr. 2008;84(2):171-7.

11. Brant TCS1, Parreira VF, Mancini MC, Becker HMG, Reis AFC, Britto RR. Breathing pattern and thoracoabdominal motion in mouth-breathing children. Rev Bras Fisioter. 2008;12(6):495-501.

12. Moreno MA, Silva E, Gonçalves M. O efeito das técnicas de facilitação neuromuscular proprioceptiva - método Kabat - nas pressões respiratórias máximas. Fisioter Mov. 2005;18(2):53-61.

13. Moreno MA, Catai AM, Teodori RM, Borges BLA, Cesar MC, Silva E. Efeito de um programa de alongamento muscular pelo método de Reeducação Postural Global sobre a força muscular respiratória e a mobilidade toracoabdominal de homens jovens sedentários. J Bras Pneumol. 2007:33(6):679-86.

14. Costa D, Gonçalves HA, Lima LP, Ike D, Cancelliero KM, Montebelo MI. New reference values for maximal respiratory pressures in the Brazilian population. J Bras Pneumol. 2010;36(3):306-312.

15. Kakisaki F, Shibuya M, Yamazaki T, Yamada M, Suzuki H, Homma I. Preliminary report on the effects of respiratory muscle stretch gymnastics on chest wall mobility in patients with chronics obstructive pulmonary disease. Respiratory Care. 1999;44(4):409-14.

16. Fauroux B. Respiratory muscle testing in children. Pediatric Respir Rev. 2003:4(3):243-9.

17. Held PA; Castro WM; Silva TLP; Silva KR; Di Lorenzo VAP. Treinamento muscular e da respiração nasal em crianças respiradoras orais. Fisioter Mov. 2008;21(4):119-27.

18. Barbiero E de F; Vanderlei LC; Neto AS; Nascimento PC. Influence of respiratory biofeedback associated to re-expansive ventilation patterns in individuals with functional mouth breathing. Int J Pediatr Otorhinolaryngol. 2008;72(11):1683-91.

19. Banzatto MG, Grumach AS, Mello JF Jr, Di Francesco RC. Adenotonsillectomy improves the strength of respiratory muscles in children with upper airway obstruction. Int J Pediatr Otorhinolaryngol. 2010;74(8):860-3

20. Takara GN, Ruas G, Pessoa BV, Jamami LK, Di Lorenzo VA, Jamami M. Comparison of five portable peak flow meters. Clinics. 2010;65(5):469-74.

21. Simon KM, Carpes MF, Imhof BV, Juk DB, Souza GC, Beckert GFQ, et al. Avaliação da mobilidade torácica em crianças saudáveis do sexo masculino pela medição do perímetro torácico. Fisioter pesqui. 2006:13(2):6-10.

22. Braz RG, Goes FPDC, Carvalho GA. Confiabilidade e validade de medidas angulares por meio do software para avaliação postural. Fisioter Mov. 2008;21(3):117-26. 
23. Baraúna MA, Deloroso MGB. Método fotogramétrico de rastreamento do ângulo de Charpy em crianças asmáticas e não asmática. Fisioter Bras. 2000;1(2):75-84.

24. Corrêa EC, Bérzin F. Mouth Breathing Syndrome: cervical muscles recruitment during nasal inspiration before and after respiratory and postural exercises on Swiss Ball. Int J Pediatr Otorhinolaryngol. 2008;72(9):1335-43.

25. Cagnie B, Danneels L, Cools A, Dickx N, Cambier D. The influence of breathing type, expiration and cervical posture on the performance of the cranio-cervical flexion test in healthy subjects. Man Ther. 2008;13(3):232-8.

26. Gaultier C. Tests of respiratory muscle function in children. Am J Respir Crit Care Med. 2002:166:601-9.
27. Pires MG, Di Francesco RC, Grumach AS, Mello Júnior JF. Avaliação da pressão inspiratória em crianças com aumento do volume de tonsilas. Rev Bras Otorrinolaringol. 2005;71(5):598-602.

28. Wilson TA, De Troyer A. Diagrammatic analysis of the respiratory action of the diaphragm. J Appl Physiol. 2009;108(2):251-5.

29. Jackson AC. Accuracy, reproducibility, and variability of portable peak flowmeters. Chest. 1995;107(3):648-51.

30. Barbiero E de F, Vanderlei LC, Nascimento PC, Costa MM, Scalabrini A. Influence of respiratory biofeedback associated with a quiet breathing pattern on the pulmonary function and habits of functional mouth breathers. Rev Bras Fisioter. 2007;11(5):347-53.

31. Corrêa ECR, Bérzin F. Temporomandibular disorder and dysfunctional breathing. Braz J Oral Sci. 2005;3(10):498-502. 